\title{
A novel fluidic oscillator incorporating step-shaped attachment walls
}

\author{
Jing-Tang Yang ${ }^{\mathrm{a}, *}$, Chi-Ko Chen ${ }^{\mathrm{a}}$, Kun-Jyh Tsai ${ }^{\mathrm{a}}$, Wei-Zhih Lin ${ }^{\mathrm{a}}$, Horn-Jiunn Sheen ${ }^{\mathrm{b}}$ \\ ${ }^{a}$ Department of Power Mechanical Engineering, National Tsing Hua University, Hisnchu 300, Taiwan \\ ${ }^{\mathrm{b}}$ Institute of Applied Mechanics, National Taiwan University, Taipei 106, Taiwan
}

Received 30 January 2005; received in revised form 6 September 2006; accepted 22 September 2006

Available online 27 October 2006

\begin{abstract}
A novel fluidic oscillator incorporating step-shaped attachment walls and acute-angle splitters has been designed and verified experimentally to stabilize the oscillation of circulation flow, to modify the flow patterns and to improve the performance. Focusing on various oscillators of plane-wall and step-wall designs, we systematically analyzed the pressure spectra, pressure loss, and flow structure, via particle-imaging velocimetry, flow visualization, and pressure sensors. The results reveal that the novel design promotes conditions to initiate the oscillator and makes the recirculation vortices oscillate more effectively and stably. The operating range of this step-wall oscillator is broadened and the ratio of signal to noise of the step oscillator is 17 times as great as that with plane walls at a greater flow rate, $65 \mathrm{~L} / \mathrm{min}$. Comparison of the wave form of the spectral analysis and the ratio of signal to noise over the entire experimental range further corroborates the superior features of the novel design.

(C) 2006 Elsevier B.V. All rights reserved.
\end{abstract}

Keywords: Fluidic oscillator; PIV; Spectra analysis; Flow structure

\section{Introduction}

A fluidic oscillator is widely applied to measure flow rates [1-3] because of a characteristic that its oscillating frequency is linearly proportional to the inlet flow rate for Reynolds numbers over a broad range. The basis of operation is that a jet entering a divergent cross-section or a sudden-expansion channel generally diverts toward either side, due to the Coanda effect, then develops to become a periodically oscillating flow at a fixed frequency [4-6]. Beyond this application in a flow meter, the oscillating features are widely used for actuators [7,8], mixers [9], and memory and control devices [10].

Previous researchers reported specific correlations among the oscillation characteristics and geometric parameters. Based on the channel structure and the operating principle, previous investigations of fluidic oscillators are classified into three types: feedback oscillator [1-3,7-9], Karman vortex oscillator [11,12], and concave-type oscillator (Vee-gutter or U concavity) [13-18]. Tippetts et al. $[19,20]$ deduced four major parameters for feedback fluidic oscillator of relaxation type, namely its Strouhal number $(S r)$, Reynolds number $(R e)$, Euler number $(E u)$, and a dimensionless control loop inductance $\left(L^{\prime}\right)$, which serve also for

\footnotetext{
* Corresponding author. Tel.: +886 3 5712916; fax: +886 35724242 .

E-mail address: jtyang@ @me.nthu.edu.tw (J.-T. Yang).
}

dimensionless analysis in discussion, defined as follows:

$$
\begin{aligned}
S r & =\frac{f h}{u} \\
R e & =\frac{u h}{v} \\
E u & =\frac{2 \Delta p}{\rho u^{2}} \\
L^{\prime} & =\frac{4 I^{\prime} n}{\pi d^{\prime 2}}
\end{aligned}
$$

in which $h$ is the characteristic length of the oscillator, $f$ the frequency of pressure fluctuation, $u$ the inlet velocity of flow, $v$ the viscosity, $\Delta p$ the pressure loss, and $\rho$ is the fluid density. Furthermore, $I^{\prime}=I / d_{n}$ and $d^{\prime}=d / d_{n}$, with $I$ and $d$ being the length and diameter of the control loop, respectively; $d_{n}$ and $n$ are the width and aspect ratio of the inlet port, respectively. Tippetts et al. further reported that for a Reynolds number smaller than a critical value no fluctuation occurred.

For a Reynolds number in a certain range, the Strouhal number remained constant; the oscillation frequency thus became linearly proportional to the flow rate and independent of fluid properties. The dimensionless control loop inductance was found to correlate linearly with the Strouhal number. Wang et al. [4-6] combined a vortex amplifier with the oscillator and 


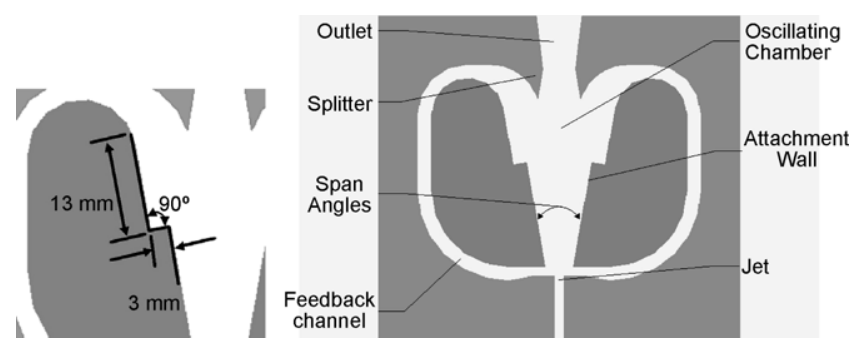

Fig. 1. Configuration of the step-wall oscillator.

significantly improved both pressure loss and oscillation spectra of an oscillatory flow meter. This design was used for remote monitoring of crude-oil pipes.

For a miniature design, Gebhard et al. used a LIGA microfabrication technique to produce a micro-oscillator of length $720 \mu \mathrm{m}$, width $500 \mu \mathrm{m}$ and depth $250 \mu \mathrm{m}$, and successfully combined this micro-oscillator with a micro-actuator to become a dynamic microsystem [7,8]. Teseř et al. [21] suggested replacing the Reynolds number with a pressure drop and derived a Teser number to characterize the micro-oscillator.

Most previous work on a fluidic oscillator emphasized more the design than an analysis of the hydrodynamic structure. In our work, we therefore undertook a quantitative analysis of the dynamic behavior of these oscillators. Based on flow phenomena, we present a novel fluidic oscillator, called a step-wall oscillator (Fig. 1) [22], and several new design concepts to improve the features of the oscillator, and seek to illuminate the design concept of fluidic oscillators.

\section{Experimental design}

The first objectives of the work are to explore the hydrodynamics in the oscillator and to investigate the function of the major fluidic elements. Then the similar and distinct fea-

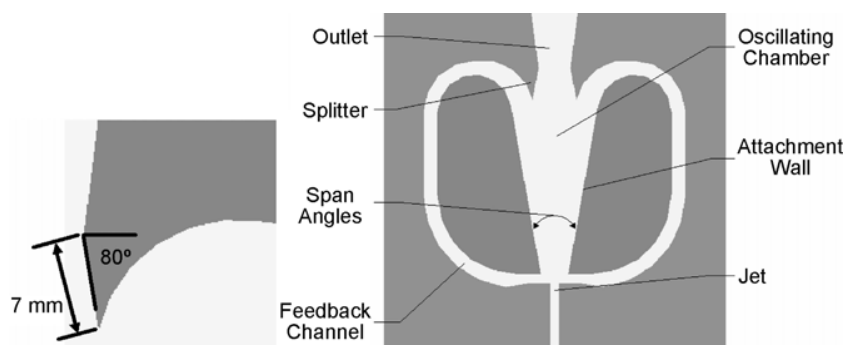

Fig. 2. Configurations of the plane-wall oscillator and splitter.

tures of the step-wall oscillator and a contrasting oscillator named a plane-wall oscillator (Fig. 2) are analyzed systematically through frequency detection and laser diagnosis. The plane-wall oscillator has been tested in our laboratory to have a superior performance among existing feedback-type fluidic oscillators.

Figs. 1 and 2 show that the fixed geometric structure has a channel of depth $40 \mathrm{~mm}$ in the entire field of the device, a nozzle $20 \mathrm{~mm}$ long and $2 \mathrm{~mm}$ wide, and feedback channels $3 \mathrm{~mm}$ wide. The detailed dimensions of the flow splitter are also marked in Fig. 2. The main control parameters are the configuration of the attachment walls, the span angle between two walls, the shape of the splitters and the flow rate. A schematic of the experimental system is depicted in Fig. 3. The investigated phenomena of oscillation dynamics comprise flow visualization of the dynamical flow structure, the correlation of spectra of pressure oscillation versus flow rate, the pressure loss, and the spatial and temporal distributions of velocity via phase-averaged particle-imaging velocimetry (PIV). Distilled water was chosen as the working substance and the diluted white watercolor, as a dye for flow visualization.

A He-Ne laser (radiant power $60 \mathrm{~mW}$ ) serves for illumination, with a semi-cylindrical lens to produce a sheet of light $1.5 \mathrm{~mm}$ thick. A high-speed video camera (Kodak KTAPRO ${ }^{\mathrm{TM}}$ )

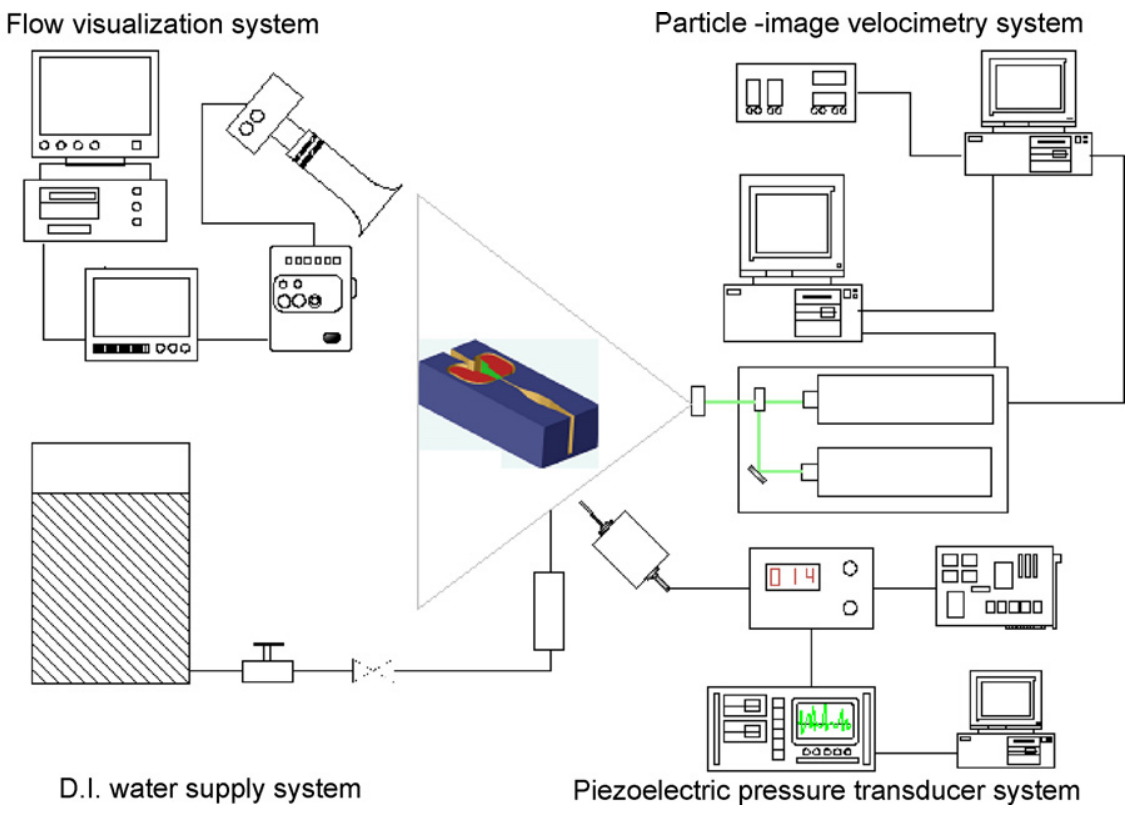

Fig. 3. Schematic of the experimental system. 
records the images of half the channel depth to obtain a twodimensional flow structure. A U-type manometer measures the pressure loss of these devices. A PIV system to measure the hydrodynamic structure combines a Nd-YAG double-pulse laser $(15 \mathrm{~Hz})$, a synchronizer, a high-resolution CCD camera and a spherical lens with a cylindrical lens, which serves to generate a thin sheet of light of thickness $106 \mu \mathrm{m}$. The high-speed video camera (TSI PIVCAM ${ }^{\mathrm{TM}} 10-30$ ) operates at 30 frames/s with resolution $1000 \times 1024$ pixels; each grid is $2.5 \times 10^{-3} \mathrm{~mm}^{2}$. The two PIV photos are separated by a $100-\mu$ s interval and calculated with a cross-correlation function. The seeding particles for PIV measurement are glass beads with diameter 37-44 $\mu \mathrm{m}$. The response time of seeding particles, $1 \mu \mathrm{s}$, is smaller than Kolmogorov's time scale, $10 \mu \mathrm{s}$; hence these seeding particles can follow the working substance and reveal the hydrodynamic characteristics. A piezoelectric pressure transducer (Kistler, type 7261) senses the pressure fluctuation in the oscillating chambers and the central feedback channels. The pressure data are analyzed via fast Fourier transform with a data analyzer (HP 35670A). The pool statistic of the pressure spectra measured in the feedback channels was $1.37 \%$ at a $95 \%$ confidence level.
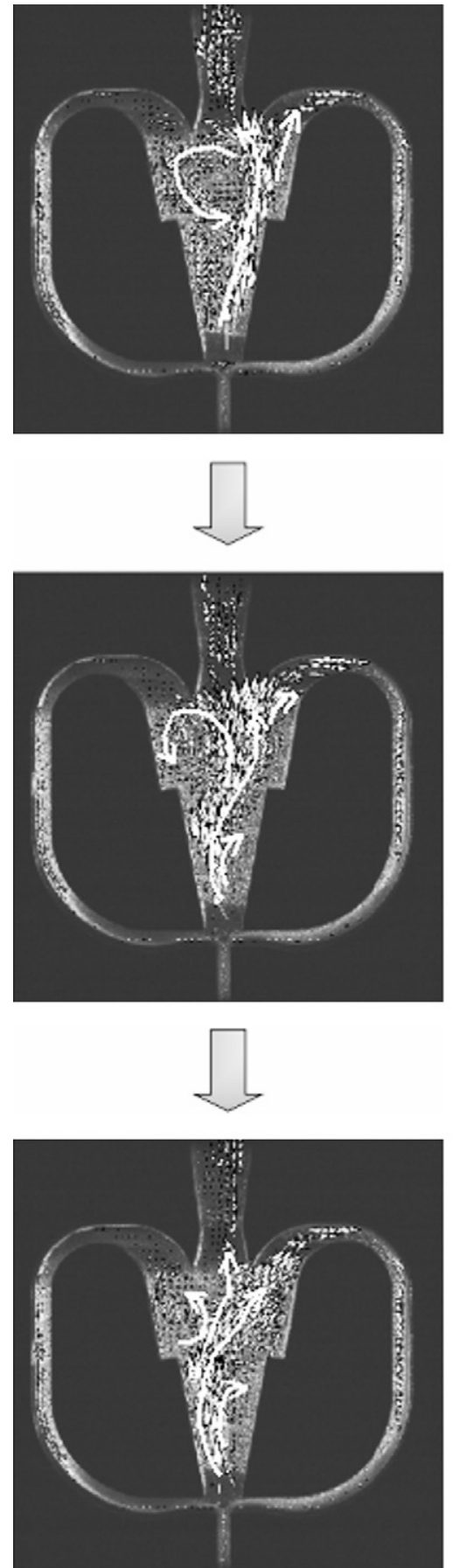
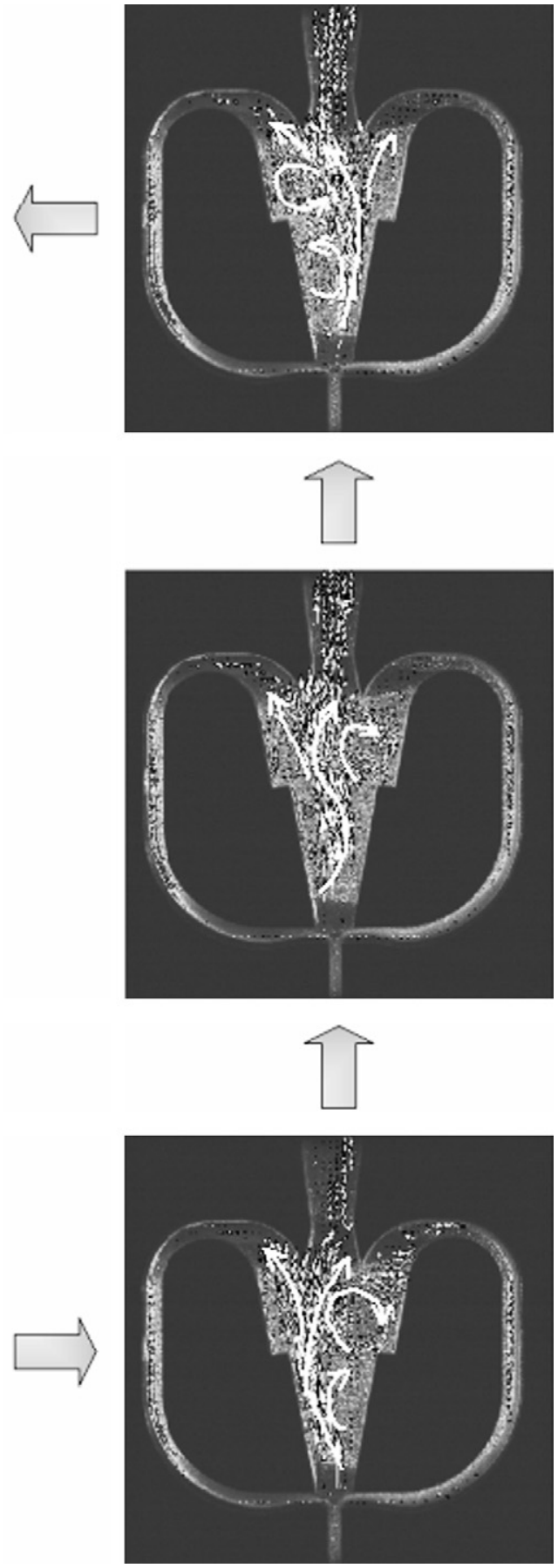

Fig. 4. Photographs and schematics of flow visualization of the step-wall oscillator. 


\section{Results and discussion}

We begin with the overall flow pattern, then contrast the performance of two geometries, and finally present our investigation of mainstream and circulation flow via PIV.

\subsection{Flow visualization}

The images of flow visualization via particle-imaging velocimetry in Fig. 4 reveal that the designed feedback channels and divergent chamber keep the mainstream oscillating periodically. The flow pattern shown in Fig. 5(a) is classifiable into three distinct regions - the inlet jet region, the vortex evolution region in the main stream, and the feedback channels, as shown in Fig. 5(b). The inlet jet entrains nearby fluid away and causes an uneven pressure gradient between the two sides of the mainstream. The uneven pressure gradient makes the mainstream oscillate transversely, so that part of the fluid in the mainstream is diverted into the feedback channel before the splitters in the downstream region. Most of the mainstream exits through the outlet port, and the evolution of vortices between splitters and attachment walls in the oscillation chamber determine the characteristics of oscillation. If the circulating vortices grow too large to retard the upstream flow, stable oscillation might be inhibited.

(a)

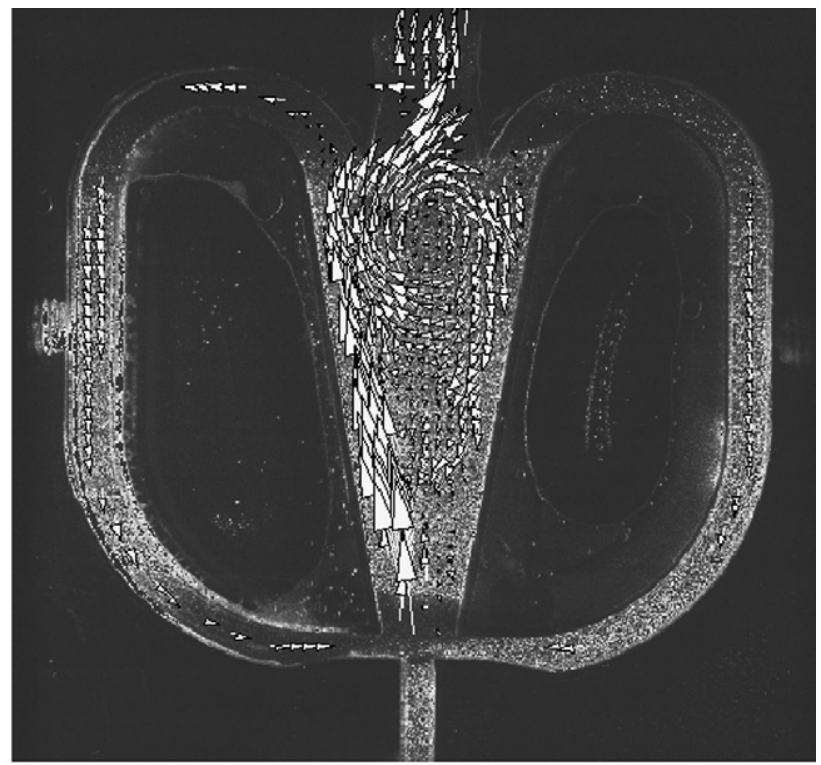

(b)
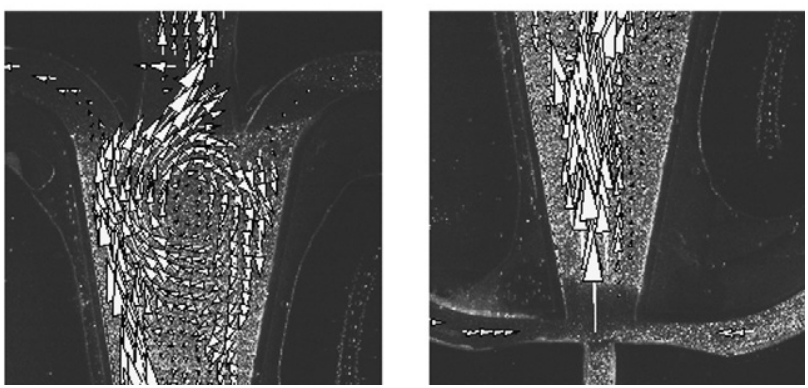

Fig. 5. PIV laser displays of fluidic oscillator: (a) flow pattern of the plane-wall oscillator and (b) local flow structures of the circulation flow and the inlet zone.
The feedback flow has a highly significant effect on the oscillator. The splitters induce feedback flow through the feedback channel that periodically impinges upstream of the mainstream. The difference between the feedback flows of the two sides exerts a transverse force and flow momentum on the mainstream. A greater rate of flow through the feedback channel strengthens the oscillation and also increases the rate of feedback flow during the next oscillation. The interactions among all three regions modulate the oscillatory characteristics.

The performance of oscillators in a series with varied parameters has been tested; the outcomes are classified as stable oscillation, unstable oscillation and no oscillation. A steady oscillation is defined as a periodic oscillation of the mainstream over a sufficiently long period. An unsteady oscillation includes two situations: one is that the mainstream tilts to one sidewall and oscillates for a while, the other is a non-periodic continuous oscillation. Fig. 6 shows the effects of span angles between the reattachment walls on the operating ranges. In these experiments, the span angle has been systematically varied from $5^{\circ}$ to $45^{\circ}$ with an interval of $5^{\circ}$. At span angle $40^{\circ}$, the circulating flow on upstream flow is more strongly inhibited than the effect of feedback flow, so that the oscillation behavior is continually unstable or even entirely quenched over the tested flow rates. An oscillating chamber with span angle $10^{\circ}$ is too narrow to provide space for stable oscillation. A span angle in a range $20-30^{\circ}$ provides the best operational design in this paper.

The angle of the flow splitter has been systematically varied from $70^{\circ}$ to $120^{\circ}$ with an interval of $10^{\circ}$ to reveal the function of the splitter on the feedback effect. In general, the splitters not only control the rates of flow diverted into the feedback channels but also manipulate the development of the circulation flow. To amplify the rate of feedback flow, we varied the angle of the flow splitter from $70^{\circ}$ to $120^{\circ}$; the results show that the pressure drag exerted on the upstream flow by the splitters also increases, which circumvents the positive effect on oscillation. The results in Fig. 7 reveal that splitters with an acute angle in a range $80-90^{\circ}$ generate the best performance. The best interaction between the span angle and the splitter angle is important for the design of a fluidic oscillator and warrants further scrutiny.

Based on images of flow visualization and the results from Figs. 6 and 7, we conclude that an optimal design of the

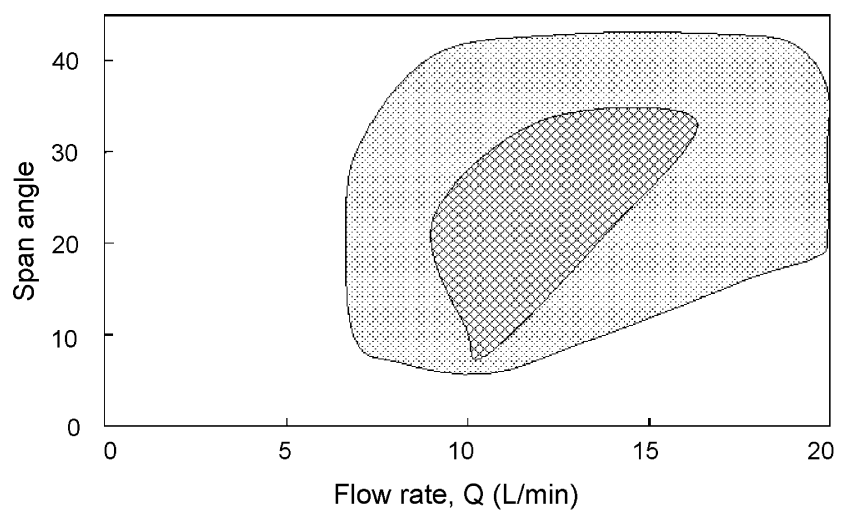

Fig. 6. Operating ranges of step-wall oscillators with various span angles: (网) stable oscillation; (圆) unstable oscillation; ( $\square$ ) no oscillation. 
Table 1

Comparison of the stable operation ranges between oscillators with plane-wall and the step-wall

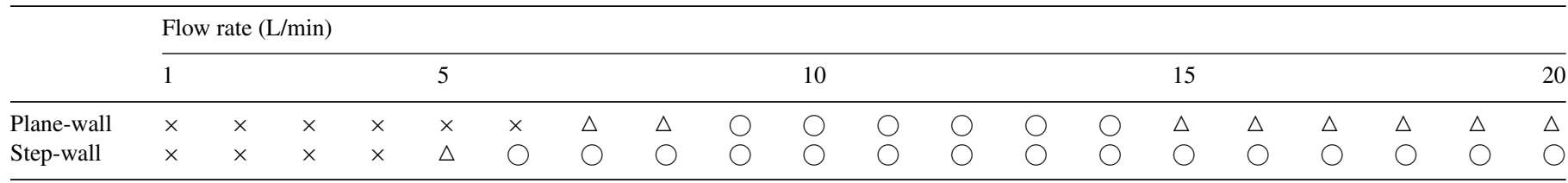

Experimental condition: 0-20 L/min. ( $\bigcirc)$ Stable oscillation; $(\triangle)$ unstable oscillation; $(\times)$ no oscillation.

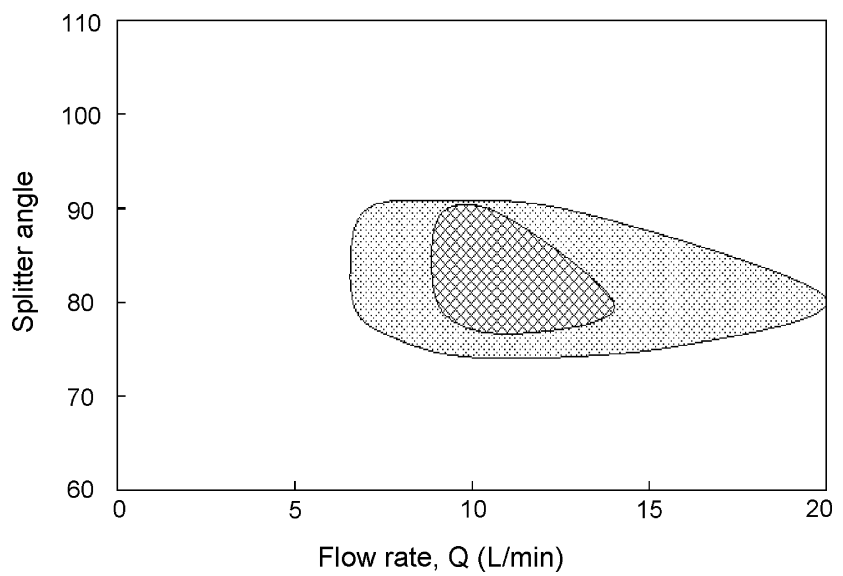

Fig. 7. Operating ranges of step-wall oscillators with various splitter angles: (ख) stable oscillation; (圈) unstable oscillation; ( $\square$ ) no oscillation.

plane-wall oscillator possesses an acute splitter angle $80^{\circ}$ and a span angle $20^{\circ}$, but the experimental results show that unstable oscillation occurs when the inlet flow rate exceeds $15 \mathrm{~L} / \mathrm{min}$, because the inhibition of circulation flow is greater than the feedback effect at large rates of flow. To improve the dynamic structure of the circulating flow, we propose a step configuration, as shown in Fig. 1, for the attachment walls; this step-wall oscillator invariably demonstrates a stable oscillation behavior in flow visualization analysis. The step structure permits an advance of stable oscillation to about $15 \mathrm{~L} / \mathrm{min}$. The contrast of operating ranges between oscillators of plane-wall and step-wall types, as shown in Table 1, proves the superiority of the proposed design. In further inquiry into the basis of the improvement, we conducted a systematic investigation as follows.

\subsection{Spectral analysis of pressure fluctuation}

The best point to undertake the spectral analysis is located in the feedback channel, as shown in Fig. 8. Because the flow pattern is simple and the interference signal generated from the complicated flow is entirely damped out there. Cascade distributions of pressure spectra for oscillators of plane-wall and step-wall types are shown in Fig. 9. The signal of the plane-wall oscillator exhibits a complicated spectral distribution and poorly distinguishes the major frequency at a great rate of flow. The waveform corresponding to the step-wall design has a distinct major frequency of oscillation with a small noise background for the flow rate over the entire range. For further identification of the pressure signals, the ratio of signal to noise (SNR) is
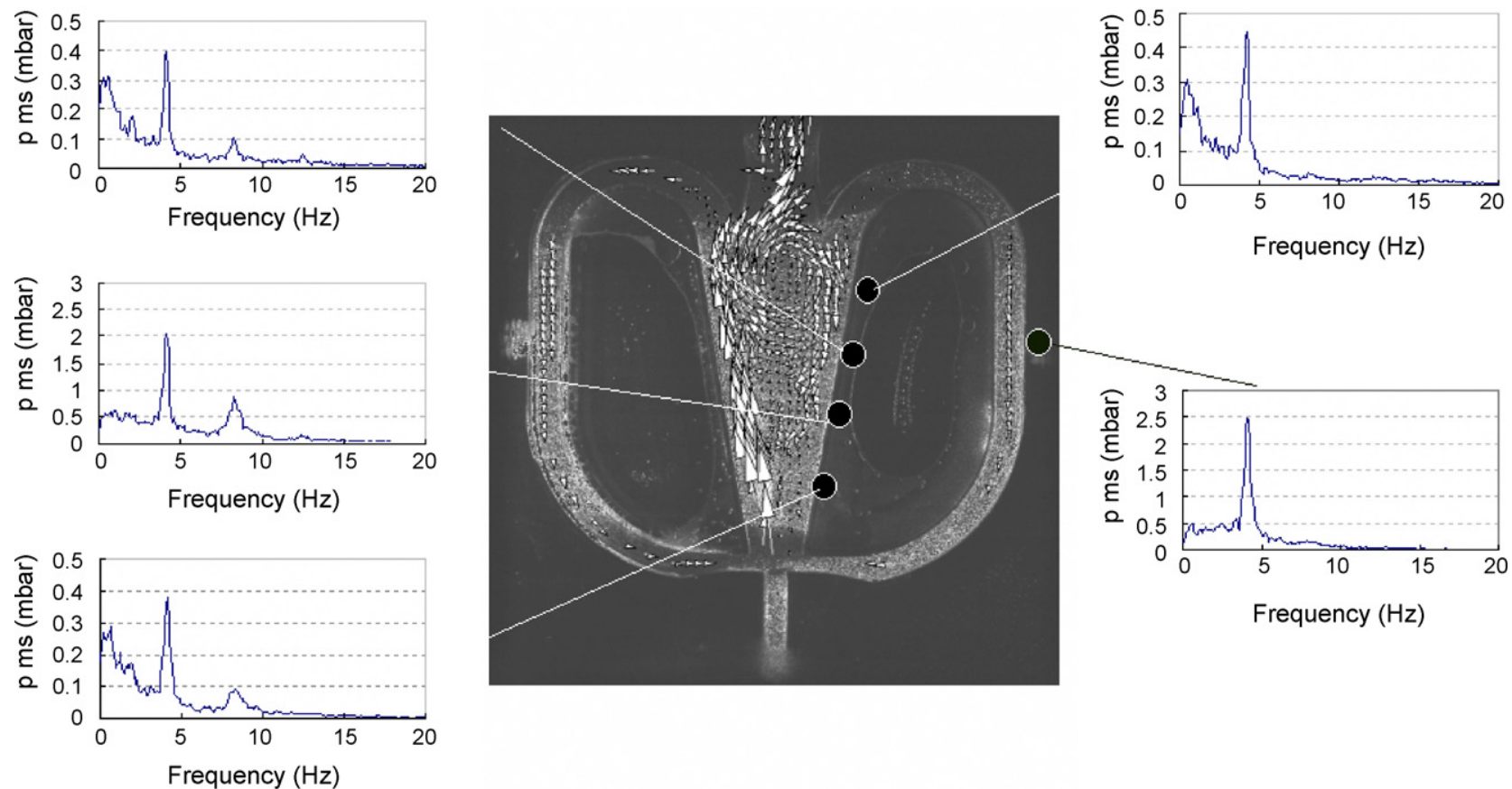

Fig. 8. Results of spectral analysis at multi-points. 

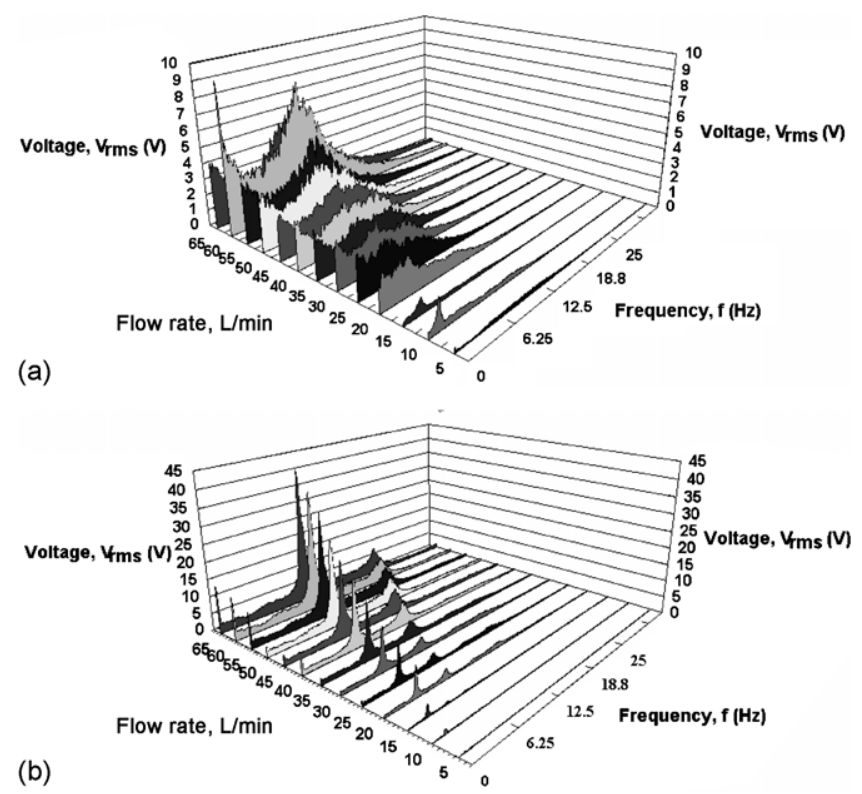

Fig. 9. Spectra cascade: (a) the plane-wall oscillator and (b) the step-wall oscillator.

introduced as follows:

$\mathrm{SNR}=\frac{V_{\mathrm{rms}}(\text { main frequency })}{V_{\mathrm{rms}}(\text { other frequecies })}$

Comparison of the SNR between the oscillators of prototype and step-wall type, as shown in Fig. 10, indicates that the step-wall oscillator induced a much greater SNR over the entire experimental range. The SNR of the step-wall design is even 17 times as great as the plane-wall oscillator $(65 \mathrm{~L} / \mathrm{min})$, which reveals that the introduction of the step configuration improves the flow pattern in the oscillating chamber, and that enhancement effect increases with increasing flow rate. The results of analysis of pressure spectra are consistent with the analysis of flow visualization.

Plots of frequency versus flow rate for oscillators of planewall and step-wall designs are shown in Fig. 11(a), indicating that the frequency of either oscillator is linearly proportional to the flow rate. The oscillation frequency of the step-wall oscillator is higher than that of the plane-wall design over the entire

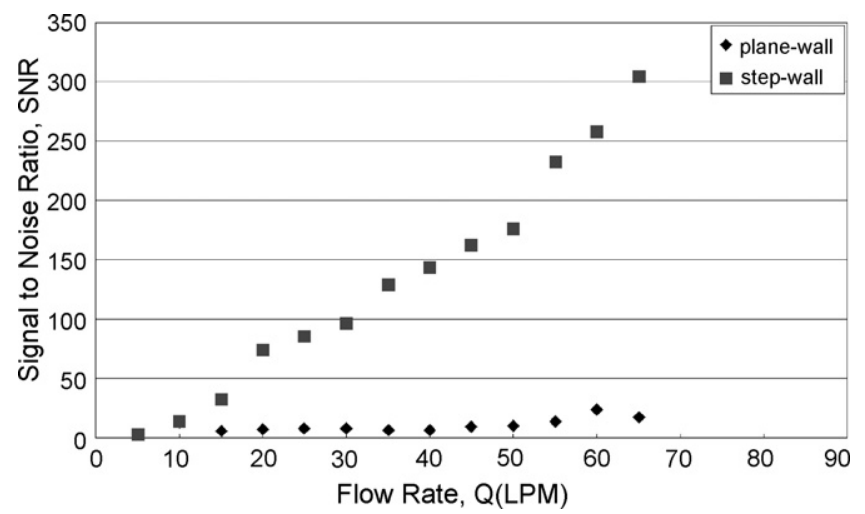

Fig. 10. Ratio of signal to noise of pressure fluctuation vs. flow rate for oscillators of plane-wall and step-wall.
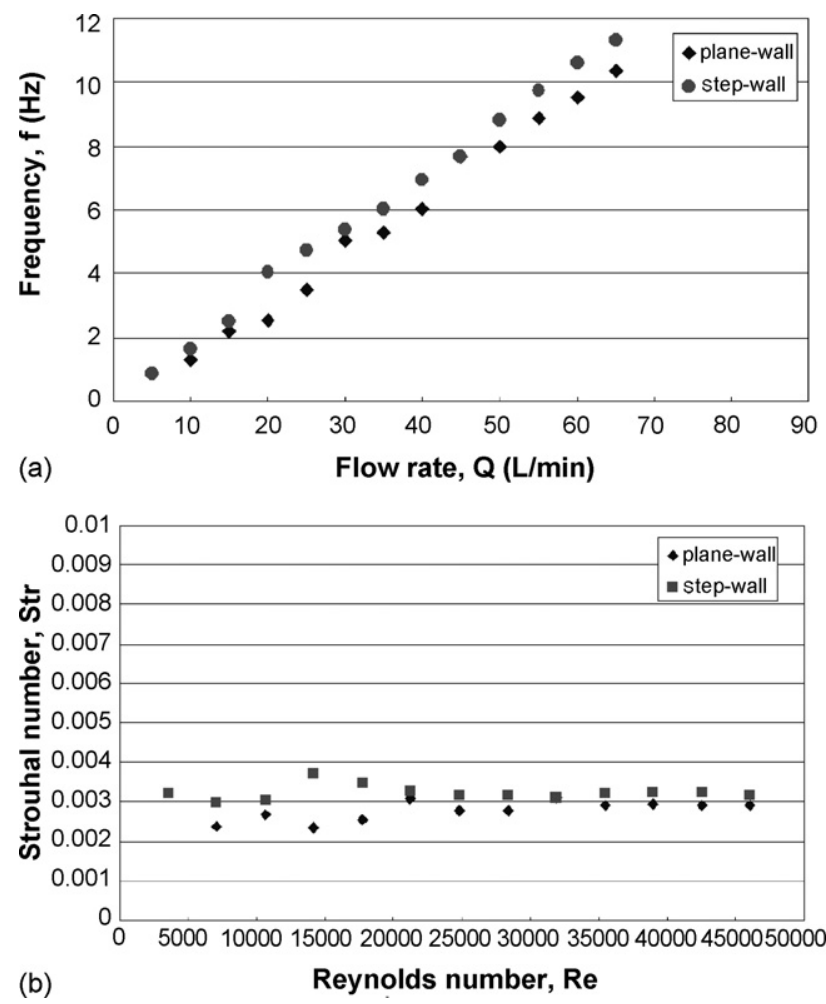

Fig. 11. Linear function for oscillators of plane-wall and step-wall operating range: (a) frequency vs. flow rate and (b) Strouhal number of pressure fluctuation vs. Reynolds number for oscillators.

operating range. An increased frequency of the step-wall oscillator indicates a smaller residence period of circulating flow and more rapid response period than those of the plane-wall design. Besides, the operating flow rate range of step-wall oscillator is broader; the smallest stable operating range is extended from 9 to $5 \mathrm{~L} / \mathrm{min}$. Fig. 11(b) shows that the Strouhal numbers of pressure fluctuations for oscillators of plane-wall and step-wall designs remain constant about $3 \times 10^{-3}$ over Reynolds number 3500-45,000.

The total pressure losses between the outlet and inlet at various rates of flow for oscillators of both types are compared in Fig. 12(a). In general, the total loss of pressure is proportional to the flow rate. The pressure loss of the step-wall design is slightly larger than that of the plane-wall type at small flow rates because the flow separates at the edge of the step. Although the sudden expansion behind the step enlarges the oscillation chamber and improves the performance, the maximum pressure loss of the step-wall design is increased up to $20 \%$ at a flow rate $65 \mathrm{~L} / \mathrm{min}$. Fig. 12(b) shows that the magnitudes of Euler number for both oscillators are similar over Reynolds numbers $8000-45,000$. The pressure loss gradually surpasses the flow inertia as the Reynolds number is decreased below 8000; the Euler number thus increases rapidly and the flow oscillation diminishes.

\subsection{Laser diagnosis via PIV}

The temporal flow structures and velocity distributions via PIV measurement for both oscillators at a flow rate $15 \mathrm{~L} / \mathrm{min}$ 

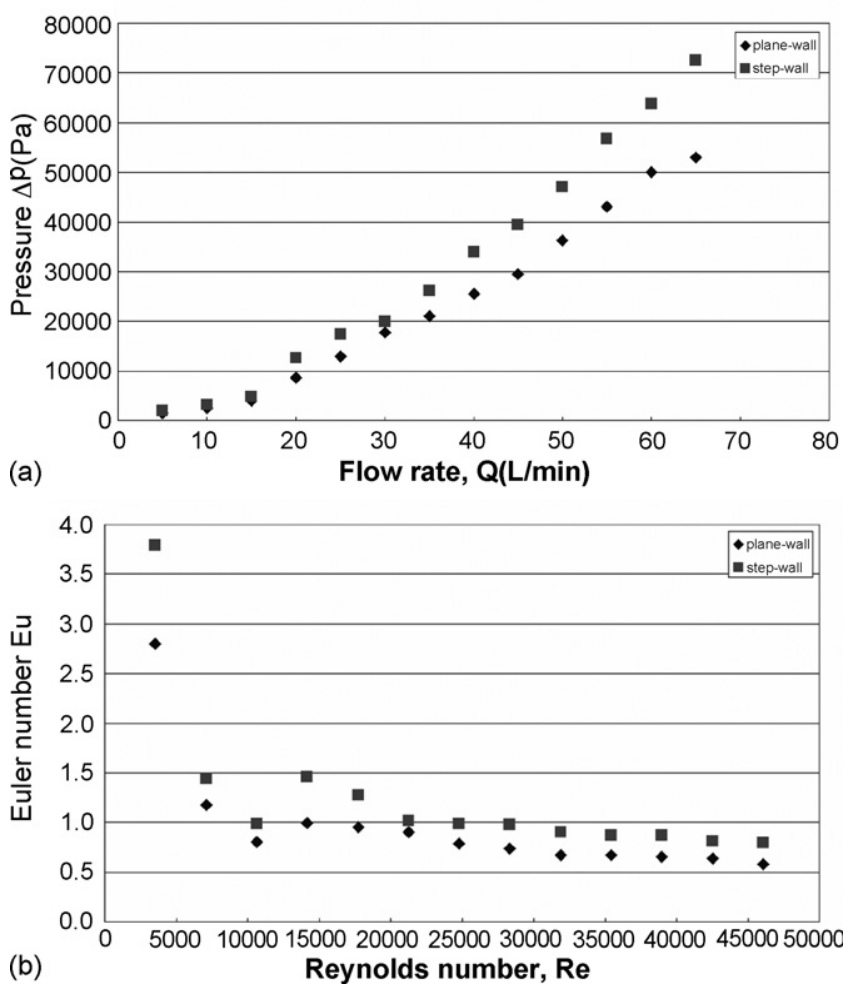

Fig. 12. The pressure drop analysis for oscillators of plane-wall and step-wall: (a) total pressure drop vs. flow rate and (b) Euler number vs. Reynolds number for oscillators.

are shown in Fig. 13. The circulation flow is larger and the circulation intensity is stronger for the plane-wall oscillator, so surpassing the excitation force in the inlet port and impeding oscillation, especially when the flow rate is small. For a large flow rate, the opposite effect occurred: the oscillation is no longer periodic. These temporal and spatial variations of hydrodynamic structure satisfactorily interpret the spectra cascades and SNR in Figs. 9 and 10. The flow structure of the step-wall oscillator is distinct from that of the plane-wall oscillator. The circulation flow originating from the separation point of the step is located between the flow splitters and steps. The circulation flow of the step-wall oscillator is invariably restrained behind the step and retains a similar size. Moreover, the step shielded the circulation flow from suppressing the upstream flow; as a result, the oscillation behavior is stable and all operating features, except the pressure loss, are notably improved.

The vertical velocity gradient across the circulation flow of the plane-wall design is stronger and the circulation intensity is greater than for the step-wall design. The differences of velocities and mass flow rates between the two feedback channels are more profound for the plane-wall oscillator than for the stepwall design. The vertical velocity gradient is also smaller for the step-wall oscillator than for the plane-wall oscillator, and the circulation zone was shielded by the step from the upstream, which facilitates initiation of oscillation. All these velocity features are consistent with the trends shown in Fig. 13.

\section{Conclusions}

In this work we investigated experimentally the flow patterns and performances of two fluidic oscillators, of plane-wall and step-wall designs, via particle-imaging velocimetry (PIV) and analysis of pressure spectra. The experimental results reveal that the dynamic behavior of an oscillator is modulated by interactions among flow fluctuations in the inlet region, the (a)

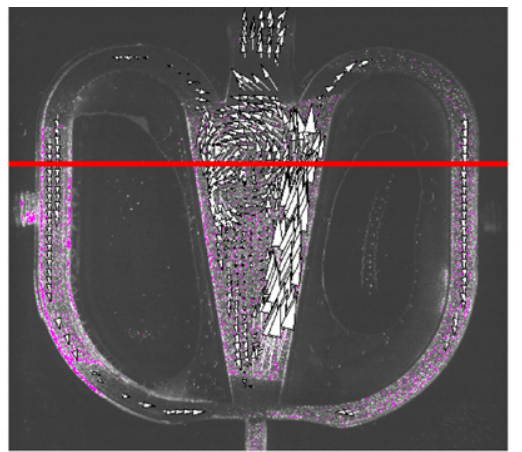

(b)

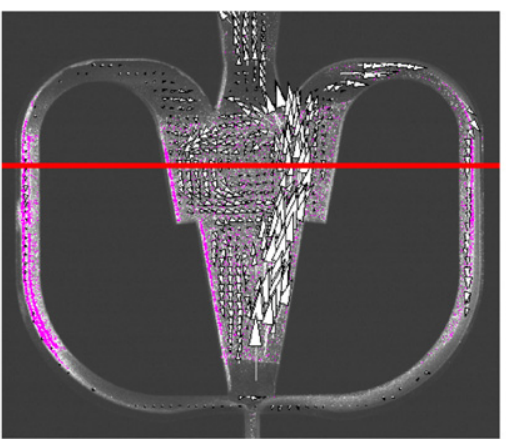

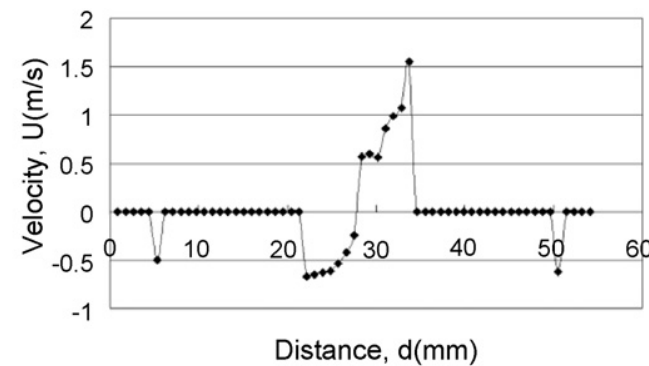

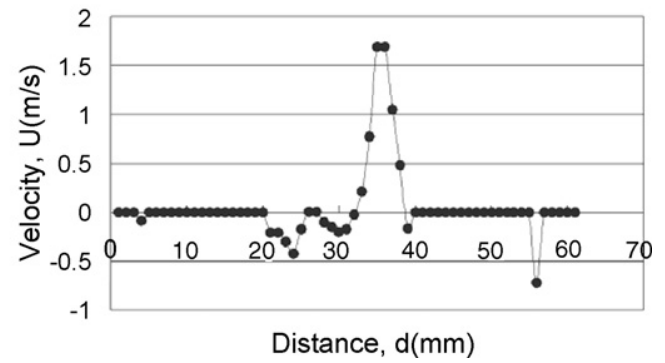

Fig. 13. Comparison of flow structure of the circulation flow quantitatively via PIV: (a) the plane-wall oscillator and (b) the step-wall oscillator. 
development of the recirculation flow, and the flow structure near step-walls and splitters. Enhancing the feedback effect and lessening the inhibition of vortex evolution and residence time improve the oscillation characteristics.

The optimal span angle between the attachment walls corresponding to the broadest stable operating range was $20-30^{\circ}$ in Reynolds number 3500-45,000. The configuration of the splitters controlled the development of the oscillating bubble and modulated the flow rate into each feedback channel and the pressure drop between the exit and inlet. Splitters with an acute angle in a range $80-90^{\circ}$ with respect to the mainstream generate the best oscillatory feature.

The modification of a step design on each attachment wall promoted conditions to initiate oscillation and made the recirculation vortices oscillate more stably between the walls. Because the mainstream flows smoothly, the recirculation intensity is also controlled better to guide part of the mainstream flow along the outer region and from the oscillator and part of the fluid into one or other feedback channel. In addition, differences of both pressure and velocity in the horizontal direction between the two feedback channels in the inlet zone are increased, thus inducing stronger and more stable periodic flow oscillation with less inhibition and a shorter stationary time of recirculation bubbles, compared with a plane-wall oscillator. The operating range of the step-wall oscillator is wider, especially down to a small flow rate $5 \mathrm{~L} / \mathrm{min}$. Besides, the ratio of signal to noise of the step oscillator was 17 times as great as that with flat walls at a greater flow rate $65 \mathrm{~L} / \mathrm{min}$. Comparison of the wave form of the spectral analysis and the ratio of signal to noise over the entire experimental range further verified the superior properties of the step-wall design.

\section{Acknowledgement}

The National Science Council of the Republic of China partially supported this work under contract numbers NSC 91-2212E-007-063 and NSC 91-2218-E-007-042.

\section{References}

[1] P.H. Wright, The Coanda Meter-a fluidic digital gas flowmeter, J. Phys E 13 (1980) 433-436.

[2] R.F. Boucher, Minimum flow optimization of fluidic flowmeters, Meas. Sci. Technol. 6 (1995) 872-879.

[3] C.K. Chen, L. Wang, J.T. Yang, L.T. Chen, Experimental and computational analysis of periodic flow structure in oscillatory gas flow meters, J. Mech. 22 (2006) 137-144.

[4] H. Wang, G.H. Priestman, S.B.M. Beck, R.F. Boucher, Development of fluidic flowmeters for monitoring crude oil production, Flow Meas. Instrum. 7 (1996) 91-101.

[5] H. Wang, S.B.M. Beck, G.H. Priestman, R.F. Boucher, Fluidic pressure pulse transmitting flowmeter, Chem. Eng. Res. Des. 75 (1997) 381391.

[6] H. Wang, S.B.M. Beck, G.H. Priestman, R.F. Boucher, A Remote measuring flow meter for petroleum and other industrial applications, Meas. Sci. Technol. 9 (1998) 779-789.

[7] U. Gebhard, H. Hein, U. Schmidt, Numerical investigation of fluidic micro-oscillators, J. Micromech. Microeng. 6 (1996) 115117.
[8] U. Gebhard, H. Hein, E. Just, P. Ruther, Combination of a fluidic microoscillator and micro-actuator in LIGA - technique for medical application, in: Proceedings of the International Conference on Solid-state Sensors and Actuators, Chicago, June, 1997, pp. 16-19.

[9] M.K. Jeon, J.H. Kim, J. Noh, S.H. Kim, H.G. Park, S.I. Woo, Design and characterization of a passive recycle micromixer, J. Micromech. Microeng. 15 (2005) 346-350.

[10] A. Groisman, M. Enzelberger, S.R. Quake, Microfluidic memory and control devices, Science 300 (2003) 955-958.

[11] T. Shakouchi, A new fluidic oscillator, flowmeter without control port and feedback loop, J. Dyn. Syst. Meas. Control. 111 (1989) 535542 .

[12] H. Yamasaki, S. Honda, A unified approach to hydrodynamic oscillator type flowmeters, J. Fluid Control. 13 (1981) 1-17.

[13] K. Yamatomoto, F. Hiroki, K. Hyode, Self-sustained oscillation phenomena of fluidic flowmeters, J. Visualization 1 (1999) 387-396.

[14] S. Honda, On the role of a target and side walls to fluidic oscillation, in: Flucome 2000, Sherbrooke (QC), Canada, August, 2000, pp. 13-17.

[15] L. Lalanne, Y. Le Guer, R. Creff, Dynamics of a bifurcating flow within an open heated cavity, Int. J. Thermal Sci. 40 (2002) 1-10.

[16] O. Uzol, O. Camci, Experimental and computational visualization and frequency measurements of the jet oscillation inside a fluidic oscillator, $\mathrm{J}$. Visualization 5 (2002) 88-96.

[17] G.B. Lee, T.Y. Kuo, W.Y. Wu, A novel micromachined flow sensor using periodic flapping motion of a planar jet impinging on a V-shaped plate, Exp. Thermal Fluid Sci. (2002) 435-444.

[18] K.T. Chang, R.F. Huang, Develop and characterization of jet-injection Veegutter, J. Mech. 20 (2004) 57-63.

[19] J.R. Tippetts, H.K. Ng, J.K. Royle, A fluidic flowmeter, Automatica 9 (1973) 35-45.

[20] J.R. Tippetts, H.K. Ng, J.K. Royle, An oscillating bistable fluid amplifier for use as a flowmeter, J. Fluid Control. 5 (1973) 28-42.

[21] V. Teseř, W.K. Allen, J.R. Tippetts, Microfluidics-the challenge of low re flow control, in: Flucome 2000, Sherbrooke (QC), Canada, August, 2000, pp. 13-17.

[22] J.T. Yang, W.Z. Lin, K.J. Tsai, K.J. Huang, Fluidic oscillator, US Patent No. 10/769,627 (January 2005).

\section{Biographies}

Jing-Tang Yang is a Professor at the Department of Power Mechanical Engineering and the Institute of Microelectromechanical Systems at National Tsing Hua University. His current research topics include microfluidics, biomimetic engineering, energy and combustion, jet propulsion, and laser diagnostics. His $\mathrm{PhD}$ degree was earned at the University of Wisconsin at Madison in August 1983. Currently, he serves as the chairman of the steering committee of Taiwan government research and development on energy, the coordinator of advanced energy research program of the National Science Council, and the director of mid-north regional center of K-12 nanotechnology education in Taiwan.

Chi-Ko Chen was born in Taiwan in 1979. He received the BS degree from Yuan-Ze University in 2001 and the MS degree in the Department of Power Mechanical Engineering from National Tsing-Hua University, Hsinchu, Taiwan, in 2003, where he is currently pursuing the $\mathrm{PhD}$ degree. His research interests include microfluidics system, $\mu$-LIF and FRET method development.

Kun-Jyh Tsai received his BS degree in mechanical engineering from National Cheng Kung University in 2001, Taiwan and MS degree in the Power Mechanical Engineering from Nation Tsing-Hua University in 2003, where he is presently a PhD student.

Wei-Zhih Lin received his BS and MS degree in the Power Mechanical Engineering from Nation Tsing-Hua University in 2000 and 2002.

Horn-Jiunn Sheen is currently a professor at the Institute of Applied Mechanics, National Taiwan University. He is also the director of the Nano-ElectroMechanical-System (NEMS) Research Center, National Taiwan University. His research interests include micro-fluidics, bio-sensors, and flow measurements. 